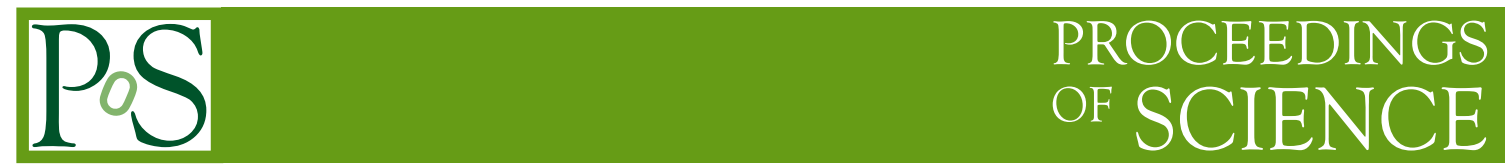

\title{
Upgrade of ATLAS Hadronic Tile Calorimeter for the High Luminosity LHC
}

\section{Ryan Mckenzie, on behalf of the ATLAS Tile Calorimeter System}

University of the Witwatersrand, Johannesburg, South Africa

E-mail: ryan.peter.mckenzie@cern.ch

The Tile Calorimeter (TileCal) is a sampling hadronic calorimeter covering the central region of the ATLAS experiment, with steel as an absorber and plastic scintillators as the active medium. The High-Luminosity phase of Large Hadron Collider (LHC), delivering five times the LHC nominal instantaneous luminosity, is expected to begin operation in 2028. The TileCal will require new electronics to meet the requirements of a $1 \mathrm{MHz}$ trigger, higher ambient radiation, and to ensure better performance under high pile-up conditions. Both the on- and off-detector TileCal electronics will be replaced during the long-shutdown of 2025-2027. Photo-multiplier signals from every TileCal cell will be digitized and sent directly to the back-end electronics, where the signals are reconstructed, stored, and sent to the Level-1 trigger at a rate of $40 \mathrm{MHz}$. This will provide better precision of the calorimeter signals used by the trigger system and will allow the development of more complex trigger algorithms. The modular front-end electronics feature radiation-tolerant commercial off-the-shelf components and redundant design to minimise single points of failure. The timing, control. and communication interface with the off-detector electronics is implemented with modern Field Programmable Gate Arrays and high-speed fibre optic links running up to $9.6 \mathrm{~Gb} / \mathrm{s}$. The TileCal upgrade program has included extensive research, development, and test-beam studies. The ongoing developments for on- and off-detector systems, as well as upcoming test-beam campaigns will be discussed.

The Ninth Annual Conference on Large Hadron Collider Physics - LHCP2021

7-12 June 2021

Online 


\section{Introduction}

In the year 2028 the start of the operation of the High-Luminosity Large Hadrdon Collider (HLLHC) is planned with a foreseen peak luminosity of $5 \times 10^{34} \mathrm{~cm}^{-2} \mathrm{~s}^{-1}$ in order to reach a final data size of $4000 \mathrm{fb}^{-1}$ by the end of its operation [1]. The resulting HL-LHC environment has necessitated the Phase-II upgrade of the ATLAS detector which is in a mature stage of development and construction [2]. The TileCal is a sampling calorimeter that forms the central section of the hadronic calorimeter of the ATLAS experiment and is located at $|\eta|<1.7 .{ }^{1}$ It performs several critical functions within ATLAS such as the measurement and reconstruction of hadrons, jets, hadronic decays of $\tau$-leptons and missing transverse energy. It also contributes to muon identification and provides inputs to the Level-1 calorimeter trigger system. The detector utilizes scintillation tiles, as the active media, which are inter-spaced by steel absorber plates. The scintillation light is readout by WaveLength Shifting (WLS) fibers and Photo-Multiplier Tubes (PMTs). These readout signals will be digitized and sent directly to the back-end electronics, where the signals are reconstructed, stored, and sent to the Level-1 trigger at a rate of $40 \mathrm{MHz}$. This will provide better precision of the calorimeter signals used by the upgraded ATLAS Trigger and Data AcQuisition (TDAQ) system and will allow for the development of more complex trigger algorithms. Due to the HL-LHCs increased luminosity, an average of up to 200 simultaneous pileup collisions are expected per bunch crossing. The new TDAQ architecture coupled with the higher radiation levels necessitates the complete redesign and replacement of the readout electronics of the TileCal. These upgrades will be accompanied by the replacement of $10 \%$ of the most exposed PMTs, the redesign of the high- and low-voltage systems as well as the mechanics.

\section{The TileCal upgrade components}

Mechanics:The TileCal is partitioned into three cylindrical barrel regions along the beam axis. These are the the Long Barrel (LB) located at $|\eta|<1.0$ and Extended Barrels (EB), of which there are two, located at $0.8<|\eta|<1.7$. Each barrel region consists of 64 wedge-shaped modules that cover the azimuthal angle $\Delta \phi \sim 0.1 \mathrm{rad}$ that are composed of plastic scintillator tiles, functioning as the active media, inter-spaced by steel absorber plates. ${ }^{1}$ An EB module is read out by 32 PMTs whereas an LB module is read out by 90 PMTs. The PMTs and on-detector electronics of a module will be housed within a new modular configuration. This configuration consists of three-meter long drawers, known as Super-Drawers (SD), which are located at the outer radius of the Tilecal. Each SD is itself composed of either four or three mechanically attached independent Mini-Drawers (MD) depending on whether they are located in the LB or EB regions, respectively. Each MD can house up to 12 PMTs. The EB modules make use of two smaller drawers in addition to 3 MDs. These smaller Micro-drawers are un-instrumented and contain up to 4 PMTs [3]. The mechanics production is in a mature state with production of the SDs approaching $\sim 50 \%$ completion.

\footnotetext{
${ }^{1}$ The ATLAS experiment makes use of a right-handed coordinate system with its origin located at the nominal interaction point (IP) in the centre of the detector and the z-axis along the beam pipe. The x-axis points from the IP to the centre of the LHC ring, and the y-axis points upwards. Cylindrical coordinates $(\mathrm{r}, \phi)$ are used in the transverse plane, where $\phi$ is defined as the azimuthal angle around the $\mathrm{z}$-axis. The pseudorapidity is defined in terms of the polar angle $\theta$ as $\eta=-\ln \tan (\theta / 2)$.
} 
Photo-Multiplier Tubes and High Voltage Active Dividers (HVADs): The PMTs, located within the SDs of a module are responsible for converting scintillation light produced in their associated TileCal cells into analog signals, which are then sent to the next stage in the signal chain. The light is transmitted from the cells to the PMTs via WLS fibres. A module is subdivided into regions, known as cells, each of which being comprised of a bundle of WLS fibres. Most cells are read out by two PMTs. A total of 768 PMTs, which are located in the most exposed regions will be replaced due to aging. An HVAD is located at the end of every PMT [4]. These divide the input HV power received from the HV-system amongst the 8 PMT dynodes. The PMTs require qualification and robustness testing before installation within the TileCal. The required test stands are fully operational. The final production of the accompanying HVADs will commence upon the completion of additional radiation studies.

Front End board for the New Infrastructure with Calibration and signal Shaping (FENICS): Located atop and attached to every HVAD is a FENICS. A FENICS is a readout electronics board that is responsible for the amplification and shaping of the analog signals received from an individual PMT. The two main roles of a FENICS are to read the fast signals using two gains and to read the average current using six gains. Pre-production of the FENICS2, the final iteration, has commenced with 40 units having been completed to date.

MainBoard (MB) and DaughterBoard (DB): A MB is located within each MD of TileCal. A single MB is connected to twelve FENICS and one DB. A MB digitizes the low- and high- gain signals received from the FENICS which it then routes to its associated DB. A MB also provides digital control of the front end boards using Field-Programmable Gate Arrays (FPGAs). The DBs are the primary high-speed interface between the on- and off-detector electronics and are mounted on top of every MB. A DB transmits detector data to the off-detector electronics, receives and distributes LHC clocks, configurations as well as slow control commands [5]. Ten V4 MBs have been installed within the TileCal and have undergone vertical slice tests with 90 units currently being manufactured. The V6 DBs are being prepared for the upcoming test-beams and require additional radiation tests before their final design review.

PreProcessor (PPr): A PPr is composed of four Compact Processing Modules (CPMs) and an Advanced Telecommunications Computing Architecture (ATCA) carrier board. Each CPM will read out and operate up to two TileCal SDs via high-speed optical links. A CPM implements a bi-directional GigaBit transceiver communication with four $9.6 \mathrm{Gbps}$ uplinks, and two $4.8 \mathrm{Gbps}$ downlinks per mini-drawer. The uplinks transmit detector and monitoring data to the CPMs. The downlinks provide Detector Control System (DCS) commands and Timing, Trigger, and Control information to the on-detector electronics, as well as the LHC clock for the sampling of the PMT signals. Deposited energy is reconstructed and calibrated in real-time using the received digitized samples. The CPM transmits this data to the trigger and data acquisition interface board via the ATCA carrier board at the LHC frequency via four 9.6 Gbps optical links. The PPr is currently in the design phase with several successful tests having occurred.

Trigger and Data AcQuisition interface (TDAQi): All interfaces between the TileCal and TDAQ are implemented in the TDAQi modules [6]. The TDAQi constructs the trigger primitives and interfaces with the trigger. It also communicates with the Front End LInk eXchange (FELIX) system which is responsible for interfacing between TileCal and the ATLAS data-acquisition system. The Trigger FPGAs compute trigger objects for electrons, jet, global, and muon triggers and the results 
are transmitted digitally through low latency high-speed optical links to the Level-0 trigger. The TDAQi V2 prototype design is currently undergoing internal review.

High-Voltage (HV) system: The HV system provides regulated high-voltage power to individual HVADs [7]. It consists of HV Remote-boards which provide primary HV received from HV Supply-boards to passive HV Bus-boards located within the Mini-drawers via long cables. The Bus-boards then distribute the power to the HVADs. The HV system has passed the majority of its preliminary design reviews with two Remote-boards having undergone combined testing with the Supplies-boards in preparation for a vertical slice test. A new manufacturer is developing an alternative HV cable to comply with strict CERN standards.

Low-Voltage (LV) system: The LV system provides low-voltage power to the front-end electronics. Off-detector AUXiliary boards provide 200 VDC to the Low-Voltage Power Supplies (LVPS) as well as performing control and monitoring functions. 256 LVPS are located on-detector each of which providing LV power to an individual module. An LVPS converts 200 VDC to the 10 VDC required by the front-end electronics using up to 8 custom electronics power converters, known as Bricks. The LV system is in an advanced state of development with many components proceeding to the pre-production phase while others await additional radiation studies.

Calibration systems: The TileCal is equipped with dedicated calibration systems that allow for monitoring and calibration of the responses of the different components of the detector. Two of the multiple TileCal calibration options are considered below. The Cesium system is used to calibrate and monitor the full optical path of TileCal. A system of $\mathrm{Cs}^{137} \gamma$-sources, driven by a liquid flow through all of the scintillating tiles, is used. New highly radiation-tolerant control boards of the $\mathrm{Cs}^{137}$ system will be implemented as well as a new optical-link interface with the DCS. The control boards are to undergo radiation tests to verify the active components selected. The Laser system makes use of an off-detector laser source that provides light to the PMTs via optical fibres. The prototype design of Interface for the LAser system to the New Acquisition infrastructure, the new control interface, is ongoing.

Upcoming Test-Beam Campaigns: The TileCal experimental setup in the H8 beam-line of the Super Proton Synchrotron consists of two fully functional LB modules, one EB module and the hardware required for their operation. Two test beams will be undertaken in September and November of 2021. The primary goal of which is to test the response of the Phase-II upgrade module readout. The following electronics are to be evaluated: The upgrade HV and LV systems, V4 MBs, V6 DBs, FENICS2, PPr and the CPM.

\section{Conclusions}

The design of ATLAS TileCal for HL-LHC is nearing completion. A large portion of the system has been prototyped and tested with some elements entering the pre-production and finalproduction stages. Additional radiation studies are being undertaken due to updated radiation policies. Beam tests are to be undertaken in September and November 2021 in order to validate the on-detector electronics in a beam environment as well as the associated off-detector electronics. The Phase-II upgrade is on schedule for installation in 2025.

Copyright 2021 CERN for the benefit of the ATLAS Collaboration. Reproduction of this article or parts of it is allowed as specified in the CC-BY-4.0 license. 


\section{References}

[1] High-Luminosity Large Hadron Collider (HL-LHC) official web page. (2020), https:// hilumilhc. web.cern.ch/content/hl-lhc-project

[2] The ATLAS Collaboration, The ATLAS Experiment at the CERN Large Hadron Collider (2008), https://doi.org/10.1088/1748-0221/3/08/s08003

[3] The ATLAS Collaboration, ATLAS-TDR-028 (2017), https://cds.cern.ch/record/ 2285583

[4] The ATLAS Collaboration, ATL-COM-TILECAL-2013-014 (2013), https://cds.cern. ch/record/1541295

[5] The ATLAS Collaboration, ATL-TILECAL-PROC-2021-011 (2021), https://cds. cern. ch/record/2783999

[6] The ATLAS Collaboration, ATL-TILECAL-PROC-2019-011 (2019), https://cds . cern. $\mathrm{ch} / \mathrm{record} / 2783999$

[7] The ATLAS Collaboration, ATL-TILECAL-PROC-2021-014 (2021), https://cds. cern. $\mathrm{ch} / \mathrm{record} / 2784250$ 